\title{
Teilnahme, Erlösung und ewiges GedenkenStrategien der Sichtbarkeit von Fürbittinschriften auf liturgischen Geräten
}

Renate Kroos zum Gedenken

Mittelalterliche vasa sacra wie non sacra, seien es Patenen, Pyxiden, Kelche oder Weihrauchfässer, tragen häufig die Namen ihrer Stifter, oftmals verbunden mit einer Anrufung Gottes, Jesu Christi oder verschiedener Heiliger. ${ }^{1}$ Im Folgenden geht es jedoch um eine Gruppe, bei denen die Stifter ,expressis verbis‘ denjenigen, welche die Gefäße in die Hand nehmen, einen Wunsch für die Zelebration der Messe mit auf den Weg geben: Dieser Wunsch kreist stets um ewiges Gedenken und Erlösung innerhalb des kontinuierlich wiederkehrenden Wunders der Wandlung. Hieraus erhebt sich die Frage nach dem etwaigen Stellvertretercharakter der Inschriften, um zum einen an der heilsspendenden Handlung auf ewig teilzuhaben und zum andern die Bitte um Vergebung und Erlösung zu verewigen. Aus dieser Frage generieren sich - gemäß des Themas des Sammelbandes - drei weitere: Muss die Sichtbarkeit der Schrift gewährleistet sein, damit die Wirkung sich entfaltet und der Adressat erreicht werden kann? Ist das Wissen um die Präsenz wichtiger als die Präsenz selbst? Oder entsteht Sichtbarkeit nur durch Präsenz?

Entsprechend der Abfolge der Wandlung der eucharistischen Gaben sei mit dem Brot begonnen, das heißt mit den Patenen und Pyxiden, gefolgt von Wasser und Wein, also den Kelchen; das Ende beschließen die Weihrauchfässer.

\section{Die Inschrift als Stellvertreterin weltlicher Stifter beim Mysterium der Wandlung}

Die älteste im Rahmen der Forschungen zum vorliegenden Beitrag entdeckte Patene ist die 1908 in Stûma (bei Aleppo) gefundene (Washington, Dumbarton Oaks Collection), die aus der zweiten Hälfte des 6. Jahrhunderts stammt und folgende griechische (hier verdeutschte) Inschrift trägt: „Für den Seelenfrieden der Sergia, (Tochter?) des Joannes, und des Theodosius, und für die Erlösung des Megalos und des Nonnous und ihrer Kinder"; ${ }^{2}$ allerdings unterbleibt die Nennung des Stifters. Dann folgt ein

1 Eine eindrucksvolle Reihe ist zusammengestellt bei Braun 1932, 171f.; des weiteren Fritz 1982, 92-94. 2 Frese 2013, 63-64, Anm. 193.

○ Open Access. (C) 2018 Johannes Tripps, publiziert von De Gruyter. (cc)BY-NC-ND Dieses Werk ist lizenziert unter der Creative Commons Attribution-NonCommercial-NoDerivatives 4.0 Lizenz. 


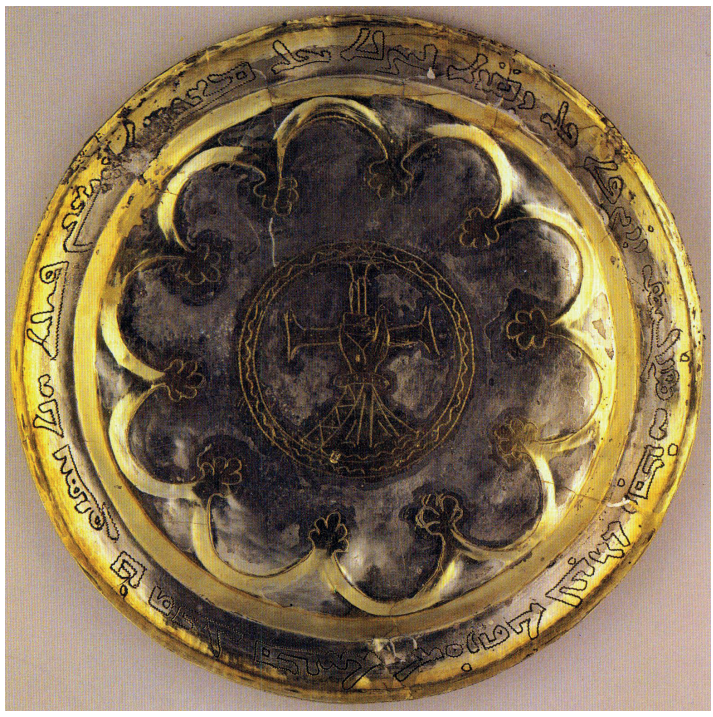

Abb. 1: Patene, um 1227. Damaskus, Nationalmuseum, Inv. Nr. 29312

Sprung von gut 700 Jahren, denn das nächste Stück ist die spätestens 1227 n. Chr. entstandene Patene aus dem Schatz von Resafa-Sergiupolis in Syrien (Abb. 1).

Die deutsche Transkription der Inschrift in Estrangela lautet: „Gestiftet hat diese Patene (der) Hasnōn, der Sohn des verstorbenen Hābēl (Abel), der Edessener, der Kirche des Mār Sergios von Resafa. Jeder, der das liest, möge für den Stifter beten. “3 Den gleichen Wunsch perpetuieren die Worte auf der Pyxis des Heinrich von Gorceke und seiner Frau Imma in der Marienkirche zu Salzwedel vom Ende des 13. Jahrhunderts (Abb. 2): ${ }^{4}$ „heinricus et vxor eius im//ma de gorceke hanc pix/idem dedervnt ecclesie sancte marie et/qui portaverit eorum memor fverit amen.“5

In ganz ähnlicher Weise bittet Propst Stephan von Sierndorf auf der von ihm nach Klosterneuburg geschenkten Patene (zwischen 1317 und 1335), man solle, so oft man dieselbe gebrauche, seiner gedenken und für seine Seelenruhe beten: „+ Stephanus ut detur requiei, quisque precetur + coelestis cena cum sumiter ista patena“. ${ }^{6}$ Und

3 Degen 1990, 68-74. Ein herzliches Dankeschön geht an Pauline Donceel-Voûte für den Hinweis auf dieses Beispiel. Die Identifikation des Schrifttyps verdanke ich Werner Arnold, Ruprecht-Karls-Universität Heidelberg, Seminar für Sprachen und Kulturen des Alten Orients - Semitistik.

4 Kruzifix auf der Deckelspitze vom Ende des 15. Jahrhunderts. Ein „Henrico de Gorceke“ wird 1280, 1287 und 1288 als einer der zwölf Ratsherren benannt; zum gesamten Sachverhalt siehe: Heidemann 2001a, 204 mit Farbabbildung auf S. 8. Fischer 1990, 80; Heidemann 2004, 120f. mit Farbabbildung; Dehio 1974, 350.

5 „Heinrich und seine Ehefrau Imma von Gorceke haben diese Hostienbüchse der Kirche der heiligen Maria verehrt und der (sie) getragen hat, möge ihrer (der Stifter) eingedenk gewesen sein. Amen“; Übersetzung nach Heidemann 2001a, 204.

6 Braun 1932, 231; Fritz 1982, 219, Abb. 242, 243. 


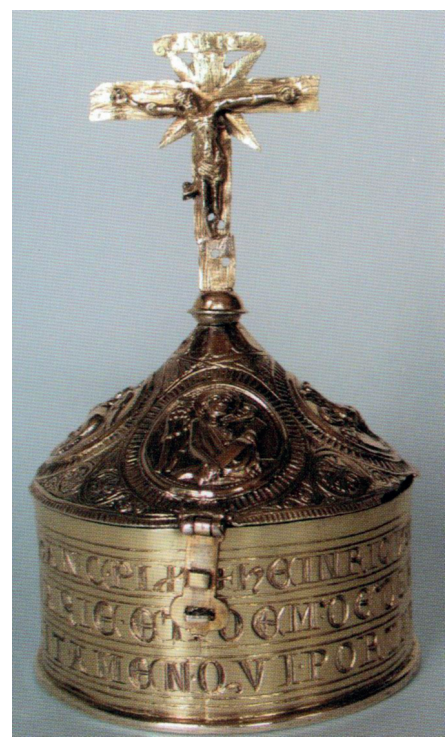

Abb. 2: Pyxis, Ende 13. Jahrhundert. Salzwedel, Marienkirche

geradezu im Sinne einer Verbrüderung mahnt die Inschrift von 1333 auf der Patene im Kopenhagener Nationalmuseum: „Hostia salutaris que hic et ubicumque pro fidelibus immolatur, R. Fratri Petro Reoneri et omnibus sibi iunctis amore seu debito speciali tam vivis quam mortuis prosit efficaciter ad veniam et salutem aeternam. Amen. 1333“. 7 Ganz anrührend lautet der Wunsch auf der Inschrift jener zwischen 1330 und 1337 in Schweden entstandenen Patene, die Ulf Abjörnesson samt Kelch zum Seelenheil seiner Gattin Christine stiftete (heute Ludwigslust, Stadtkirche): „†Ob Patris aeterni decus Wlpho, natus Aberni - Vas pro Christina dedit hoc, cui sit medicina Gratia larga Dei, statuens animam requiei“. Ulf Abjörnsson war ein mächtiger Mann in Schweden: Ritter, Reichsrat und Lagmann in Tiohärad. Am 29. September 1330 gibt er dem Pfarrer Nils in Dagsberg (Östergötland) ein Grundstück samt Vergünstigungen unter der Bedingung, dass Seelmessen für ihn und genannte Ehefrau Christina gelesen werden. 1337 vermählt sich Ulf erneut. Das legt den Schluss nahe, dass seine erste Gattin 1330 verstarb. ${ }^{8}$

$\mathrm{Zu}$ all den in den vergangenen Abschnitten aufgezählten Wünschen tritt aber noch eine ganz andere, strategische Intention von Seiten weltlicher Stifter, geboren aus dem seit dem 4. Jahrhundert schwelenden Konflikt zwischen Klerus und Laien infolge des Ausschlusses der letzteren von der Wandlung bzw. eucharistischen Feier durch Velen; nur zu ganz bestimmten Momenten gaben sie den Blick auf das

7 Braun 1932, 231.

8 Braun 1932, 231. Andersson, I, 1956, 38f., 164-167, 197-202; Andersson, II, 1956, 29f., Nrn. 45 und 46, zwischen 1330 und 1337 entstanden, Fritz 1982, 209-210, Abb. 187-188. 
Geschehen am Altar frei; somit fühlten sich die Laien in die unbefriedigende Rolle des Zuschauers abgedrängt. ${ }^{9}$ Dieser Wunsch nach dem Partizipieren erhielt in der Hoch- und Spätgotik durch das Vierte Laterankonzil 1215 mit dem Dogma der Realpräsenz sowie mit der Einführung des Fronleichnamsfestes 1264 heftig Nahrung. ${ }^{10}$ Doch sollen die Quellen sprechen, denn ihre Deutlichkeit erübrigt jede weitere Interpretation. 1505 klagt der französische Augustinereremit Johann Bechoffen in seiner Messerklärung: „Männer wie Frauen kommen viel zu nahe an die Altäre heran. Frech schauen sie den zelebrierenden Priestern ins Gesicht, wahrhaftig mehr aus verwegener Neugier als aus Andacht, und das ist sehr tadelnswert. " ${ }^{11}$ In gleicher Weise mahnt ein Grafitto an der Chorwand der Stiftkirche von Sankt Goar: „Hinaus mit Euch, Ihr Laien! Hier ist nicht Euer Ort. Hier sollen vielmehr die Freunde des Altares stehen. "12 1473 erlässt der Ulmer Rat eine ähnliche Mahnung und 1507 der Rat von Heilbronn (Neckar). ${ }^{13}$ Das bedeutet: Wenn es sich um Inschriften von Laienstiftern auf Patenen wie Pyxiden handelt, dann perpetuieren diese nicht nur den Wunsch nach Erlösung, sondern ermöglichen auch infolge der Namensnennung des Wohltäters demselben eine unmittelbare Teilnahme am Mysterium der Eucharistiefeier, von der er leiblich ausgeschlossen bleibt. ${ }^{14}$ Die Sichtbarkeit der Schrift gewährleistet somit dessen Präsenz.

Die nicht zu übersehenden Inschriften auf den beiden genannten Patenen wie der Pyxis garantieren des Weiteren, dass Priester und Diakon sie wahrnehmen; darüber hinaus befinden sie sich an Stellen, die die Zelebrierenden während des Ritus mit den Fingern unweigerlich berühren. Die Sichtbarkeit der Inschrift samt ihrer Position gewährleistet somit das Erreichen der Adressaten.

In fast identischer Weise kehrt diese Idee von schriftlich perpetuiertem Stifterwunsch und Berührung durch die Zelebranten viele Jahre später auf der Pyxis (um 1458) des Heinrich Brumzele im Osnabrücker Domschatz wieder. Die Mahnung der Inschrift an die Priester lautet: „Hinricvs bruzel decretorv $(\mathrm{m})$ doctor ac rector primi altaris me fieri fecit orate pro eo“. ${ }^{15}$

9 Frese 2013, 100-102.

10 Tripps 2000², 21f., 136, 144f., 150, 217, 221, 236.

11 ,viri quibus mulieres nimis altaribus propinqunt, in faciem celebrantium audacter prospiciunt verius ex praesumptione aut curiositate, quibus ex deuotione quod est ...”; Text wie Übersetzung zitiert nach Habenicht 2016, 142, Anm. 545.

12 „Ite foras layici, non est vester locus yci, sed stab[unt?] yci, qui sunt altaris amici.”; Text wie Übersetzung zitiert nach Habenicht 2016, 142, Anm. 546.

13 Habenicht 2016, 143.

14 Zur Gegenwart Christi bei der Messe siehe Frese 2013, 110-120.

15 „Heinrich Brumzele, Doktor des Kirchenrechts und Rektor des ersten Altares, hat mich machen lassen, betet für ihn“; siehe Fritz 1982, 309, 340, Abbildung 884. 


\section{Inschrift und Transzendenz}

Einen Schritt weiter unter den Vorzeichen von „Sichtbarkeit - Adressat - Wirkung“ geht die 1394 datierte Hostienmonstranz (Köln?) zu St. Peter und Paul in Ratingen, denn hier ist die Gruppe der Adressaten infolge der Nutzung einer eucharistischen Monstranz zum sakramentalen Segen, zu Prozessionen und vor allem zur Aussetzung des Allerheiligsten zur Anbetung durch die Laien noch größer, da schon der Anblick der geweihten Hostie als heilbringend galt. Eine Predigt aus der Zeit des beginnenden 14. Jahrhunderts beschreibt, dass das Schauen des Leibes des Herren eine Veränderung der Seele des Schauenden bewirke, denn dieser werde daraufhin schwach für sinnliche Lüste und stark an der Seele zur Überwindung der Gefahren; durch die Schau des heiligen Leibes werde nicht nur die Seele hell und klar, sondern sinnliche Begierden und weltliche Lüste besiegt, um endlich die Zunge zum Lob Gottes erheben zu können. ${ }^{16}$ Am Treffendsten formuliert Roger Bacon in seinem opus maius diesen heilsbedeutsamen Vorgang während des Sehens: Wie das Licht von einem Sehobjekt direkt auf den „Kristall“ im Auge fällt, so trifft die Gnade Gottes unreflektiert und ungebrochen die menschliche Seele. ${ }^{17}$ Die auf der Ratinger Monstranz eingravierten Worte, in diesem Falle auf Deutsch, perpetuieren damit einen Wunsch, der mit dem Anblick der Hostie und dem von ihr ausgehenden divinum lumen, wie Witelo es nennt, ${ }^{18}$ unmerklich in die Seele des Betrachters fällt: „bid vor den priester de dit cleynoyt al up geven heet - deser synre kyrken to ratinghen ter eren des heylgen sacraments anno dm m ccc xc iiii“. Gemeint ist der damals amtierende Pfarrer Bruno Meens. ${ }^{19}$

Aber Bruno Meens hat mit seinem Wunsch um Fürbitte in Anwesenheit der Eucharistie noch eine ganz andere Intention, die keiner besser formuliert als der 1230 verstorbene Pariser Theologe Wilhelm von Auxerre in seiner Summa aurea: „Die Gebete vieler werden beim Anschauen des Fronleichnams erhört, und vielen wird Gnade eingeflößt.“20

Die Lesbarkeit der Schrift muss somit also nicht unbedingt gewährleistet sein, damit die Wirkung sich entfalte und der Adressat erreicht wird. Ein ähnliches Beispiel ist für London verbürgt. 1507 bekräftigt Sir Stephen Peacocke in seinem letzten Willen eine Schenkung an die Kirche von St. Martin-next-Ludgate: „oon pix or monstre of silver and gilte, to be delivered ymmediately after my decesse. And the same there to

16 Laabs 2000, 93, 95. Codex Graecensis 730, Unibibl. Graz Bl. 194b-196a; zit. nach Franz 1902, 101.

17 Bergdolt 1988, IL.

18 Bergdolt 1988, LXV.

19 Der Stifter ist der aus Duisburg stammende Bruno Meens, Pfarrer in Ratingen (seit 1371) und Kanonikus in Xanten; er trat 1398 in die Kölner Kartause ein; sämtliche Informationen nach Fritz 1982, 243, 343, Abbildungen 409-411.

20 „Multorum petitiones exaudiuntur in ipsa visione Corporis Chr., unde multis infunditur gratia“; Sachverhalt und lateinisches Zitat nach Browe 1933, 55. 
be contynued and used to honour and lawde of almighty God as long as the same will thereunto endure". ${ }^{21}$

Der obere Teil der „pix or monstre“ mit dem eucharistischen Behältnis hat die Reformation nicht überlebt, jedoch der Sechspassfuß samt Nodus und Manschetten, mutierte er doch mit einer Cuppa versehen zum Messkelch (Ashmolean Museum, Oxford, Leihgabe des Corpus Christi College). Der Fuß trägt Londoner Hallmarks für den Zeitraum 1507/8 und die Meistermarke „H with I placed across it fesswise like a fret“. ${ }^{22}$ Bis heute schwingt sich die originale Inschrift den Sechspass des Fußes entlang und verrät den wahren Sachverhalt: „praye for the salle of stewyn pekoc and margret hys wyff wyche gave thys in the wvssheppe of the sacrement" ${ }^{23}$

\section{Heiliger Leib, Fegefeuer und inschriftliche Bitte}

Weshalb gerade das Brot so sehr mit Bitten verbunden wurde, erklären Predigten und Tafelbilder. Bereits eine Predigt des 13. Jahrhunderts verwendet das Evangelium vom 7. Sonntag nach Pfingsten und das Wort des Herrn „Misereor super turbam“, um die Barmherzigkeit Gottes gegen die armen Seelen zu preisen:

\footnotetext{
Gott erweise jenen Seelen, die lange im Fegefeuer leiden müssten, seine Barmherzigkeit, indem er unsere Gebete, Almosen und Fasten für sie annähme, ihnen Engel zur Tröstung sende und sie mit seinem heiligen Leibe erquicke; wie Gott einst dem frommen Daniel Speise in die Löwengrube sandte, so sende er den leidenden Seelen seinen heiligen Leib in der Messe, wenn der Priester die heilige Hostie in drei Teile breche, so lege er zwei Teile für sich und die Gemeinde auf die Patene, den dritten Teil lege er jedoch in den Kelch für die Seelen im Fegefeuer. ${ }^{24}$
}

Diese Vorstellungen behalten ihre Gültigkeit bis zur Reformation, wie Retabelfragmente belegen. Kommen wir zunächst zu zwei um 1525 gemalten Tafeln aus dem Programm der Werktagseite des Altares eines Anonymus im Tiroler Landesmuseum in Innsbruck (Abb. 3).

Man sieht im oberen Bildfeld des rechten Flügels einen Priester, der den dritten Teil der konsekrierten Hostie für die armen Seelen im Fegefeuer in einem Kelch deponiert; im unteren bringen die Engel dann - genau wie in der Predigt geschildert - den Leib des Herrn hinab zu den Gepeinigten. ${ }^{25}$ Ein weiteres Beispiel bildet das um 1480 entstandene Retabel aus St. Pölten (Privatbesitz): Hier sind es jedoch Strahlen, die von der vom Priester elevierten Hostie ausgehen und hinab ins Fegefeuer fallen, um die, welche dort schmachten, zu erlösen. ${ }^{26}$

21 Der gesamte Sachverhalt geschildert nach Jackson, I, 1967, 151.

22 Zitat nach Jackson, I, 1967, 151.

23 Jackson, I, 1967, 151.

24 Franz 1902, 240f. mit Anmerkung 5; Grieshaber 1844, 78 f.

25 Jezler/Konrad 1994, $290 \mathrm{f}$.

26 Jezler 1994, 23 mit Abbildung 13. 

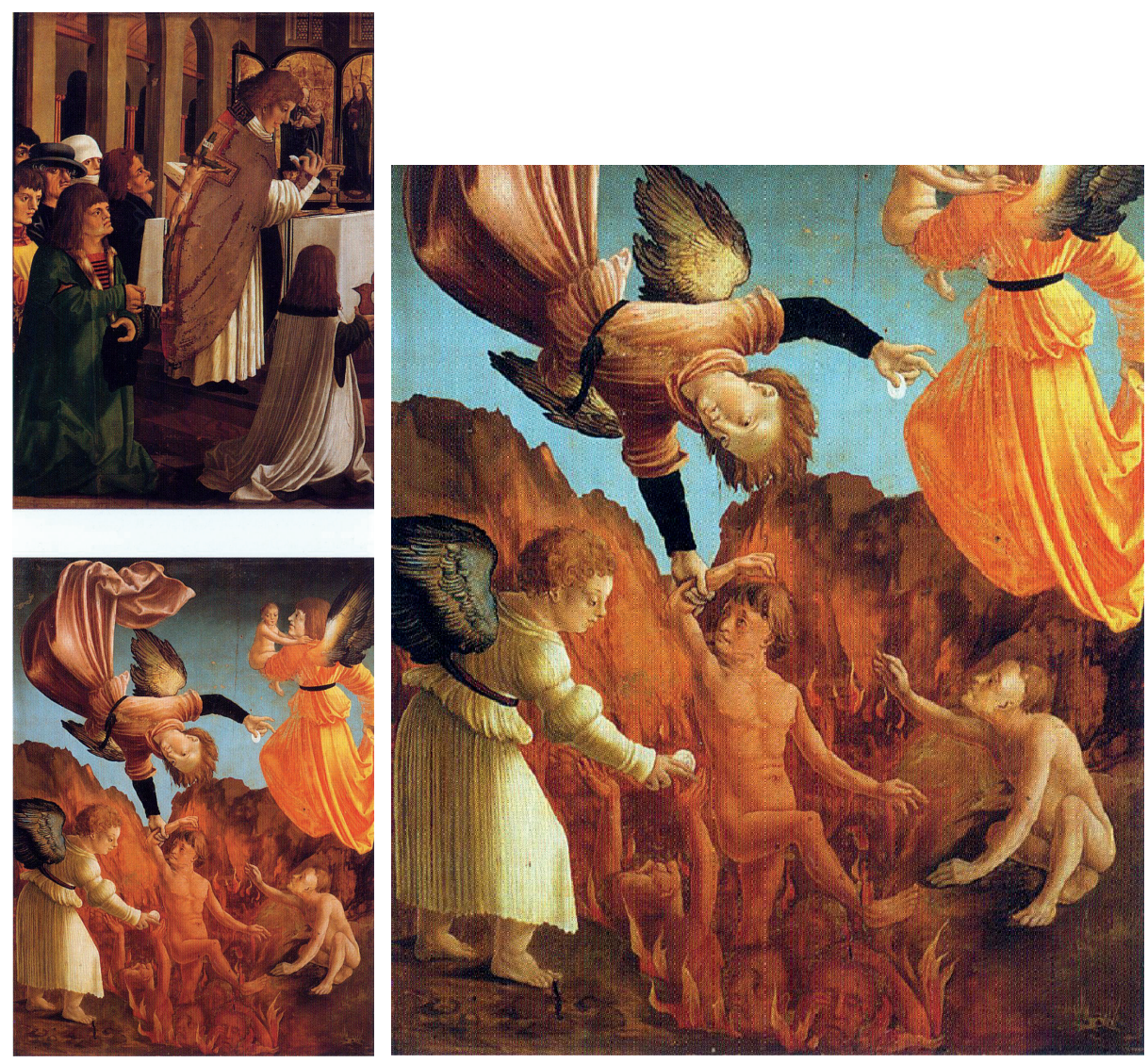

Abb. 3: Zwei Tafeln aus der Werktagsseite des Retabels der Michaelskapelle zu Niederolang (ehemals), Pustertal, um 1525. Innsbruck, Tiroler Landesmuseum

\section{Präsenz rangiert vor Lesbarkeit: die Position von Fürbittinschriften auf Kelchen}

Die Inschriften auf Kelchen spiegeln in puncto Hoffnungen prima vista einen nahezu identischen Sachverhalt wie jene auf Pyxiden und Patenen wider, indem sie ganz persönliche Botschaften der Stifter an die Zelebranten übermitteln; jedoch sind diese Botschaften mitnichten derart leicht zu entdecken bzw. zu erfassen wie jene auf den Patenen und Pyxiden, denn sie stehen entweder auf den Zargen der Kelche oder laufen an den Rändern der Füße entlang; manchmal sind sie sogar in die Unterseite des Standringes eingraviert und damit dem Blick bis zur Spülung des Gefäßes völlig entzogen. Weshalb die Präsenz des Stifters bei der Wandlung durch die stellvertre- 
tende Inschrift mit dem Wunsch auf Erlösung noch vor der Lesbarkeit der Bitte rangiert, erklären zwei Revelationes der heiligen Birgitta von Schweden.

In Kapitel 56 (1344/1346) ihrer Relevationes extravagantes sieht Birgitta ihren verstorbenen Gatten Ulf Gudmarsson im Fegefeuer schmoren, der ihr ans Herz legt, sie möge doch Messkelche stiften für das heilige Opfer, denn diese seien besonders geeignet, die Zeit der armen Seelen im Fegefeuer zu verkürzen. ${ }^{27}$ In Kapitel 9 (1350er Jahre) des Vierten Buches ihrer Revelationes kommt der gottvergessene Nicola Acciajuoli, einst Großseneschall von Neapel, angesichts der Schwere seines Sündenregisters nicht so leicht davon; um dessen Leidenszeit zu verkürzen, sollen seine Nachfahren nämlich dreißig Kelche für neunhundert Seelmessen stiften. Ein Engel erklärt Birgitta die Anzahl: Ein jeder der dreißig Priester soll mit seinem Kelch jeweils neun Seelmessen zu Ehren der Märtyrer, neun zu Ehren der Bekenner und neun zu Ehren aller Heiligen zelebrieren; dagegen sei die achtundzwanzigste Messe als Engelmesse zu lesen, die neunundzwanzigste zu Ehren der heiligen Maria und die dreißigste zu Ehren der Heiligen Dreifaltigkeit. ${ }^{28}$

Im Rahmen der vorliegenden Forschungen kam für das 13. Jahrhundert nur ein Kelch mit einer Bittinschrift ans Licht: das um 1228 getriebene Prachtstück des Hugo von Oignies im Schatz der Schwestern von Notre-Dame zu Namur (Belgien; Abb. 4).

Die Inschrift auf des Kelches Zarge lautet: „HUGO ME FECIT: ORATE PRO EO: CALIX ECCLESIE BEATI NICHOLAI DE OIGNIES: AVE“. ${ }^{29}$

Um sich zu vergegenwärtigen, welche Zeremonie ein Messkelch zu Zeiten Hugos beim eucharistischen Opfer durchlief, wie oft ihn Priester und Diakon ergriffen, verehrten und damit die fürbittenden Inschriften wahrnahmen, sei aus den Pastoralanweisung des 1296 verstorbenen Durandus von Mende zitiert, die er den Priestern seiner Diözese an die Hand gab:

Bei den Worten 'accipite et bibite' hält der Priester den Kelch etwas hoch und sagt dabei die Worte 'in remissionem peccatorum'. Dann stellt er ihn auf den Altar zurück und betet ihn mit einer ehrfürchtigen Verneigung an. Wo zwei Korporalien im Gebrauch sind, bedeckt er oder der Diakon den Kelch mit einem derselben und hält ihn so andächtig in die Höhe, so dass er vom Volke gesehen und angebetet werden kann. Dann stellt er ihn mit den Worten 'hec quociescunque feceritis etc.' vorsichtig zurück, faltet die Hände und betet ihn mit einer ehrfürchtigen Verneigung an. Dabei kann er, wenn er will, das Gebet sprechen 'ave, sanguis Christi, qui effusus es pro redemptione generis humani`. Diejenigen aber, die nur ein Korporale benützen, heben den Kelch unbedeckt empor und decken ihn, gleich nachdem sie ihn zurückgestellt haben, wieder $\mathrm{zu}^{30}$

27 Birgitta 2015, 276-278.

28 Birgitta 2008, 43.

29 Skubiszewski 2003, 108-123; Braun 1932, 172.

30 Zitiert nach Browe 1933, 40. 


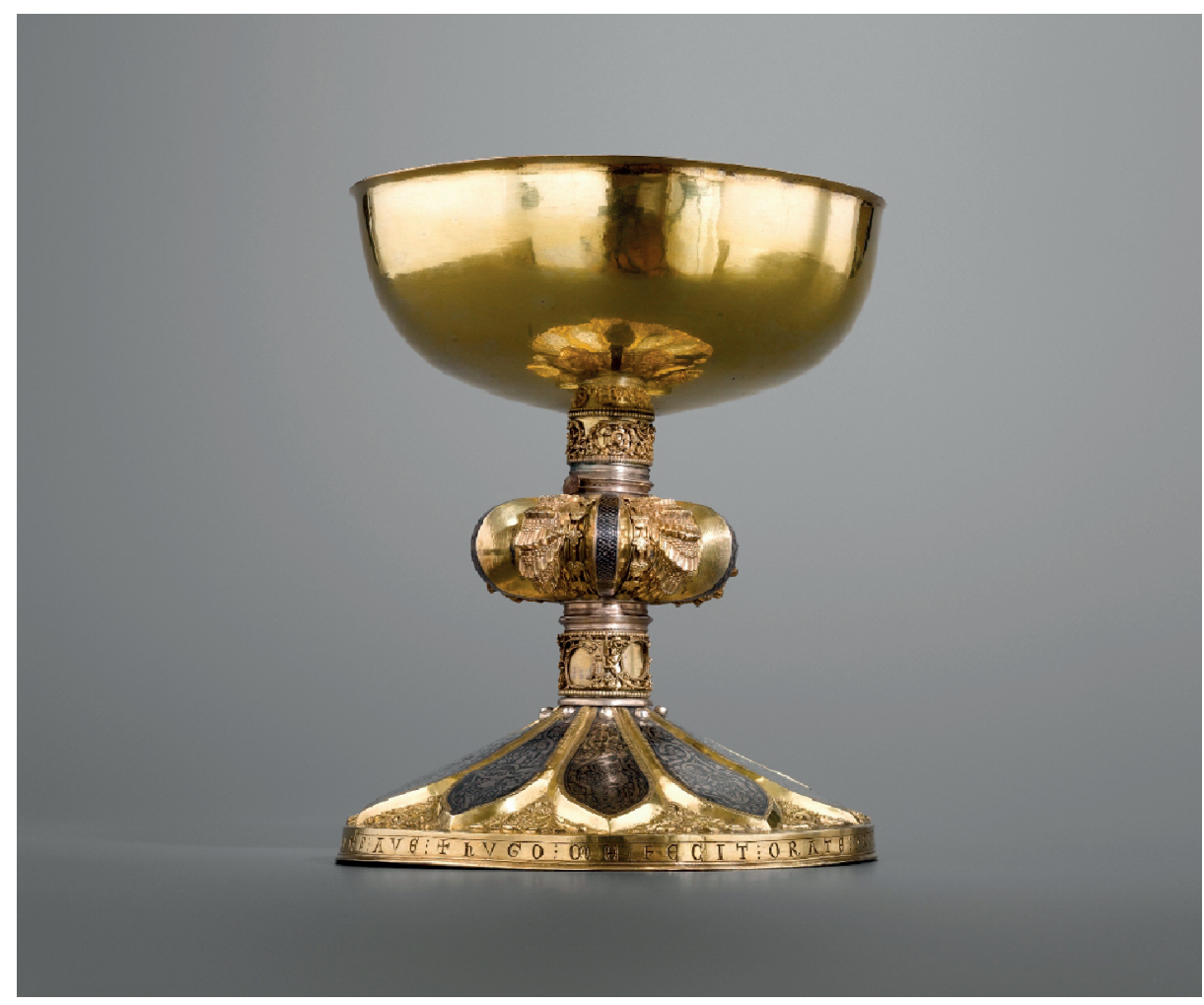

Abb. 4: Hugo von Oignies, Meßkelch, um 1228. Namur, Couvent des Sœurs de Notre-Dame, Schatzkammer

Gehen wir mit diesem Wissen ins 14. Jahrhundert. Besonders aufschlussreich an Fürbittinschriften sind hier schwedische Kelche. Da sie bislang in der Forschung ein ziemliches Schattendasein führen, sei im vorliegenden Beitrag die Gelegenheit ergriffen, sie ins Bewusstsein zu rücken. Die Reihe eröffnet die Bitte auf dem Kelch der Kirche zu Södebykarl (Uppland), die die Eltern des Stifters miteinschließt: „: EGO : PETRUS : PRESBITER : DE : RINGBOHÆREDI : FILIUS : ELAVI : ET : GERPRUDIS : DE : SAMØ : DEDI : ISTUM : CALICEM : PRO : ANIMA : PARENTI : MEORUM : PREDICTORUM““. ${ }^{31}$ Ein Pfarrer Petrus ist im ersten Jahrzehnt des 14. Jahrhunderts nachweisbar. ${ }^{32}$ Zitierter Inschrift folgt diejenige auf dem Kelch zu Ramsta (Uppland): „IN : NŌIE : DNNI : NICOLA : DE KORSHAMM : ET VXOR : CI : INGRIDIS : DEDERUV[N] $\mathrm{T}$ (und in der inneren Reihe) ME : ECCCE : BEATE : VIRGINIS : VPSALIE : PRO : A AIAB :

31 Andersson, II, 1956, 42, Nr. 72, Taf. 25.

32 Andersson, I, 1956, 36-37, 61, 81, 86, 91, 109, 112f., 125, 137f., 147, 171f., 218. 
SVIS. “33 Es handelt sich um den 1335 verstorbenen Nicolaus von Korsham, der in der Liebfrauenkirche zu Uppsala bestattet war, wohin er auch den Kelch gestiftet hatte. ${ }^{34}$

Zeitlich schließt sich - wir wechseln das Land - die Inschrift des Kelches (nach 1350) aus Gröpelingen (Bremen, Fockemuseum, Inv. Nr. 5378), gestiftet von Heinrich Groning, 1351 bis 1367 als Senator in Bremen nachweisbar: „orate hinric groning et vxor ei alheydis““. ${ }^{35}$ Es folgt die Mahnung auf dem Kelch zu Dörnhagen bei Paderborn, gestiftet von Bischof Heinrich Spiegel zum Desenberg (1360-1380): „Qui panem vite tractas cum sangue vite - Presulis Henrici recolens sis mente fideli.“36

Einen bislang einzigartigen Fall stellt die Seelmeßstiftung des 1380 verstorbenen Karls V. von Frankreich zu St.-Denis dar; der König schenkte einen Kelch samt zweier Leuchter, doch waren es die Füße dieser Leuchter, welche laut Inventar von 1505 jeweils folgende Inschrift trugen, die nicht nur ihren Stifter nannte, sondern auch die Anzahl der täglich für den Monarchen zu singenden Seelmessen einforderte: „Je fuz donné par le roy Charles, filz du roy de France Jehan, en sa chapelle, qui est fondée en l’honneur de sainct Jehan dedans de l'église Sainct Denis, où chacun jour ordinairement doivent pour luy chanter deux messes à tousjours perpetuellement." ${ }^{37}$ Die Sorge schien berechtigt, denn ganz ähnlich mahnen die eingravierten Worte auf dem Fuß eines Kelches (spätes 14. Jahrhundert) der ehemaligen Zisterzienserklosterkirche zu Dargun in Mecklenburg: „Gheze ghaf dessen kellik on der scal me alle daghe mede misse holden, dat er god gnedich sy“.38

Zeitlich schließt sich der rein goldene und um 1390 entstandene Kelch aus Arasløv (in Øster Gøngo Herred/Skåne) im Kopenhagener Nationalmuseum an, gestiftet von Christine Pettersdotter (Galen). Er trägt die Inschrift: „Domina Cristina de Arvaslef ad honorem Dei pro refrigerio animarum carorum suorum hunc calicem aureum fieri fecit. Orate Dominum pro eis“. ${ }^{39}$

Ihm folgt der um 1400 getriebene Kelch der Flensburger Marienkirche mit seiner deutschen Inschrift: „bischopp eckel van ripen hevt ghegheven dissen kelck bidet vor $\sin \mathrm{s}(\mathrm{eel}) “{ }^{40}$

Die zunehmende Betrachtung der Gleichwertigkeit der Elevation von Hostie und Kelch im 14. und 15. Jahrhundert hatte offensichtlich zur Folge, dass der Kelch mit

33 Andersson, II, 1956, 35, Nr. 58, Taf. 62-63.

34 Andersson, I, 1956, 38, 63, 87, 94, 108, 112f., 144, 196f., 205, 239.

35 Fritz 1966, 145 mit Abbildung 116; 147, 459 mit Katalognummer 106.

36 „So oft man mit dem Kelche die Messe feiere, möge man, bittet Bischof Heinrich, treuen Sinnes seiner gedenken.“; siehe Braun 1932, 172.

37 Braun 1932, 171; hier jedoch der Sachverhalt aufgrund des summarischen Schatzinventares von 1739 irrtümlich als Kelchinschrift angegeben. Im ausführlichen Inventar von 1505 der korrekte Sachverhalt samt Inschrift detailreich geschildert; siehe darum Omont 1901, 197 (Angaben im Inventar von 1505); 209 (Angaben im Inventar von 1739).

38 Kratzke 2004, 67, 340f.; Braun 1932, 171; Schlie, I, 1896, 550 mit Abbildung.

39 Sämtliche Informationen nach Fritz 1982, 255, 341, Abb. 493.

40 Fritz 1982, 255, 343, Abb. 494. 
dem Blute Christi mit der Gabe der Hostie an die zu erlösenden Seelen gleichzieht. ${ }^{41}$ Kein zweites Thema zeigt genanntes Gleichziehen der eucharistischen Gaben bei der Erlösung der armen Seelen eindrücklicher, als die sich seit dem Ende des 14. Jahrhunderts sprunghaft verbreitende Darstellung der Legende von der Messe des heiligen Gregor. Sie findet während der zweiten Hälfte des 15. und im ersten Drittel des 16. Jahrhunderts ihren Höhepunkt.

Im Folgenden zwei Beispiele, die das Gesagte im Bilde zeigen: In der Darstellung der Gregorsmesse auf dem Sippenaltar des Meisters von 1473 in St. Maria zur Wiese in Soest (1473) steht der Schmerzensmann auf dem Altar hinter Kelch, Patene, Messbuch und Leuchter (Abb. 5).

Aus seinen fünf Wundmalen spritzt das Blut zunächst in den Kelch auf der Mensa und dann vom Kelch in weiten Bögen in Richtung Kirchhof auf die dort aus ihren Gräbern Steigenden; ${ }^{42}$ hinsichtlich des Bezuges zur Errettung der armen Seelen aus den Fegefeuer gibt die Tafel des Meisters des Lebensbrunnens das Thema noch klarer wieder (um 1510; Kreuzlingen, Sammlung Heinz Kisters; Abb. 6).43

Hier springt aus der Seitenwunde Christi ein Strahl in den Kelch auf dem Altar und ein weiterer Strahl fährt hinab in den Höllenrachen, der sich neben dem Stipes weit aufsperrt, und erlöst die armen Seelen.

Es kommt einem unweigerlich die 1364 reich an Pfründen ausgestattete Seelmeßstiftung des Ritters Konrad von Wolfurt für die beim Kloster Pfäfers gelegene Kapelle der heiligen Maria Magdalena in den Sinn. Konrad tätigt dies alles - so die Stiftungsurkunde - aus großer Reue ,necnon pro salute anime mee, progenitorum meorum et omnium eorum, quos in persona vel rebus unquam letaliter vel eciam alias quomodocumque offendi.... “. ${ }^{44}$ Der Kelch, älter als das Datum der Stiftung, trägt an der Zarge umlaufend eine angesichts des Inhaltes der Urkunde geradezu nüchterne Inschrift, denn es unterbleibt jegliche Bitte um ein ewiges Gedenken, geschweige denn um die Rettung der Seele Konrads: „CVNRADUS DE WOLFURT MILES VIRGINI MARIE HYNC CALICEM DONAVIT““. ${ }^{45}$ Es ist die übliche, auf Kelchen häufig anzutreffende Formel. ${ }^{46}$

41 Zur Gleichwertigkeit der Elevation von Hostie und Kelch im 14. und 15. Jahrhundert siehe Browe 1933, 39-48.

42 Gärtner 2007, 140f. mit Abb. 4.

43 Gärtner 2007, 125 mit Abbildung 1; Kisters 1994, 294 mit Abbildungen auf S. 293 und 295.

44 „für das Heil meiner Seele, für das Seelenheil meiner Vorfahren und für das Seelenheil aller derjenigen, die von mir an Leib und Gut, tödlich oder auf irgendeine andere Weise verletzt worden sind“; das Original befindet sich im Stiftsarchiv Pfäfers (im Stiftsarchiv St.Gallen), Archivsignatur , „StiAPf, Urk. 20.09.1364“; Zugang unter http://scope.stiftsarchiv.sg.ch/detail.aspx?id=290; mein herzlicher Dank für die Informationen geht an Dr. phil. Jakob Kuratli Hüeblin, MAS, Amtsleiter-Stv./Stiftsarchivar-Stv.

45 Lanz 1994, 222f.

46 Zahlreiche Beispiele zusammengestellt durch Elbern 1963, 8, 18, $123 f$. 


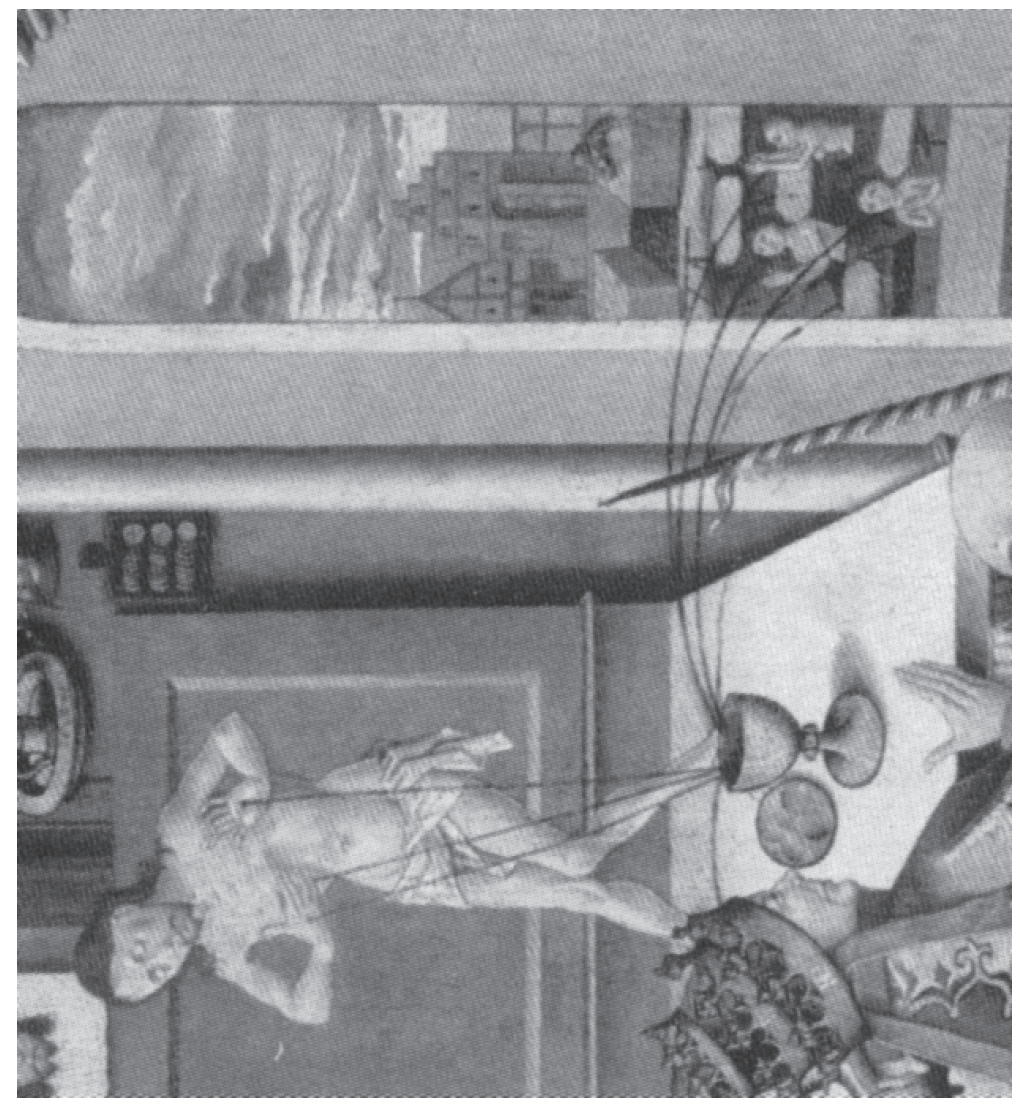

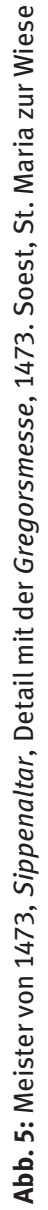



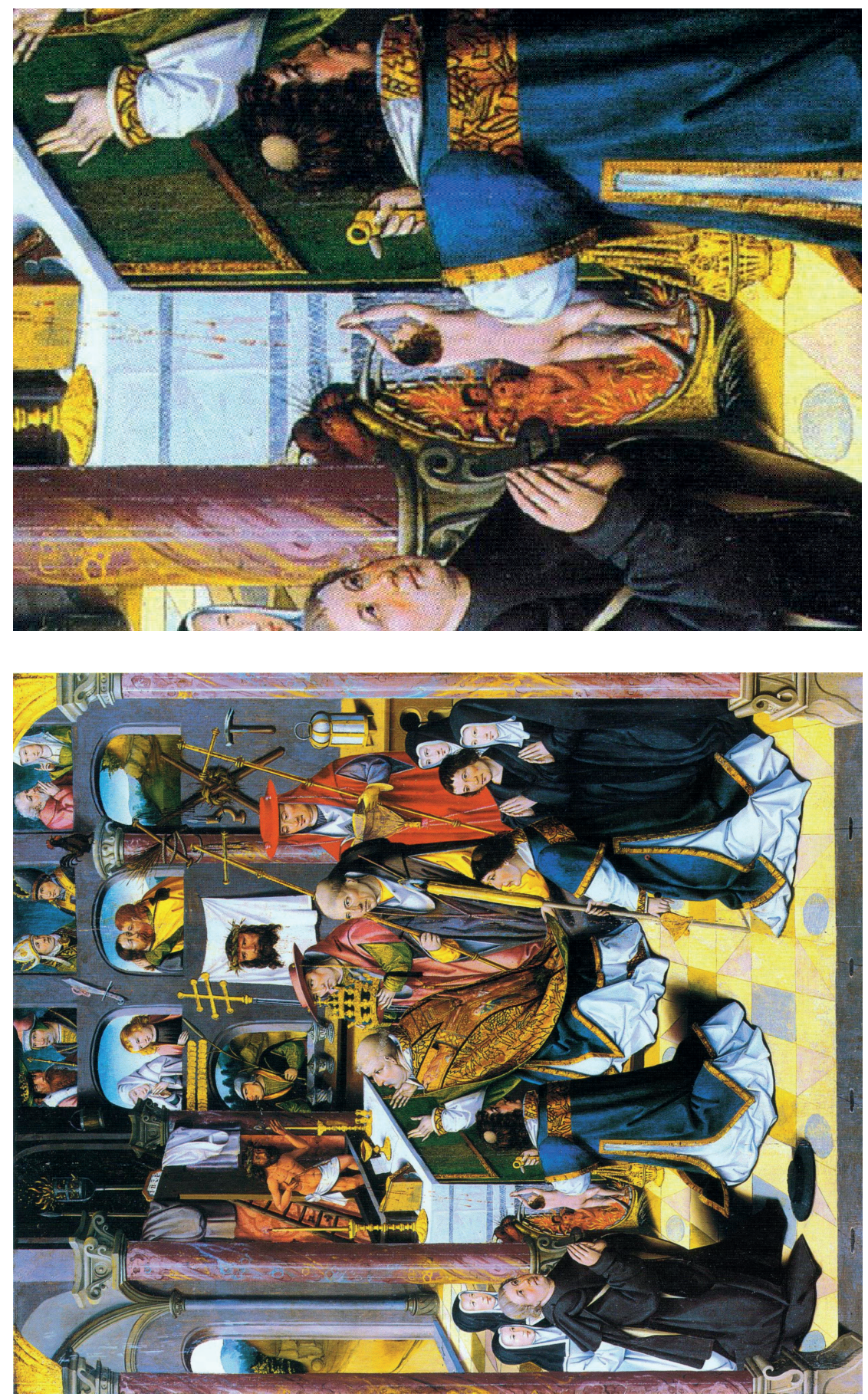

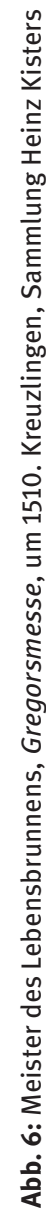


Dagegen bittet die Inschrift des Kelches in der Kirche zu Askeby (Östergötland): „oretur pro tordone bonde ro iikeson milite qui fecit hunc calicem parari ad honorem beate birgitte“; ;7 es handelt sich um den 1417 verstorbenen und in Kloster Vadstena beigesetzten Ritter Thord Rörikson Bonde. ${ }^{48}$ Dagegen schließen die Bitten des folgenden Kelches wieder die Vorfahren mit ein: Auf dem Exemplar der Riddarholmkirche zu Stockholm heißt es: „tēa kalkin latar sistr tala tidhimās dott gora i klara klostar for sic oc for sina foraldra siäla“; ${ }^{49}$ leider lässt sich die Klarissin Tala Tidhimansdotter, die den Kelch ins Stockholmer Klarenkloster gab, nicht genauer fassen. ${ }^{50}$

Ein bislang einmaliger Fall ist jener des Goldschmieds Heinrich Horne, denn er gab gleich zwei Kelchen seinen Namen sowie seine Bitten mit auf den Weg und sicherte sich so doppelte Hilfe:

Zunächst auf dem Kelch (Anfang 15. Jahrhundert, Medaillons 2. Viertel 14. Jahrhundert) der Marienkirche zu Gardelegen; die unten, in die Standfläche des Fußes eingravierte Inschrift appelliert: „orate pro hinriko horne aurifabro“. ${ }^{51}$ Hier ist der Goldschmied offensichtlich der Stifter.

Dann - ganz anders - auf dem Kelch (Anfang 15. Jahrhundert) aus dem Kloster Althaldensleben heute zu Kloster Neuendorf, seinerzeit gestiftet von Berta von Alvensleven (Abb. 7). Die Inschrift schmiegt sich rundumlaufend den Vierpaßschwüngen des Fusses an: „hu(n)c calice(m) de(di)t berta // rel(i)c(t)a gevehardi de // alvev(n)sleve ad clavstr(vm) // nie(n)dorp p(ro) memoria.“

Dreht man den Kelch aber um, dann erscheint - verborgen auf dessen Standfläche - eine zweite Inschrift: Gleichsam im Huckepack bittet „vt llj yeghet henryko horne / factor yst / orate p(ro) eo“.52

Scheinbar hatte die Witwe Berta von Alvensleben als Stifterin des Kelches nichts dagegen, dass ihr Goldschmied Heinrich Horne hier parallel zu ihr für sein eigenes Seelenheil Sorge trug. So anekdotenhaft, ja amüsant das Ganze prima vista anmutet, es kann schwerlich ohne Wissen Bertas geschehen sein und damit dürfte es sich um ein Zeichen der Wertschätzung von Seiten der Auftraggeberin an den Goldschmied handeln; genau wie einst 1311, als die Sienesen es Duccio gestatteten, sich auf der Maestà des Sieneser Domes mit folgender Bitte zu verewigen „MATER SCA DEI.SIS CAUSA SENIS REQUIEI.SIS DUCIO VITA.TE QUIA PINXIT ITA“. ${ }^{53}$ Das Retabel wird nota bene - am Fronleichnamsfest des Jahres 1311 geweiht. $^{54}$

47 Andersson, II, 1956, 10, Nr. 4, Taf. 111.

48 Andersson, I, 1956, 40, 69f., 93, 210f., 239.

49 Andersson, II, 1956, 36, Nr. 61, Taf. 111.

50 Anderson, I, 1956, 40, 66, 210f., 218, 230, 239.

51 Heidemann 2001b, 256-258; Dehio 1974, 102; Parisius/Brinkmann 1897, 75f.

52 Andrae 2001, 258f.; Andrae 2004, 146-148 mit 3 Farbabbildungen; Dehio 1974, 233; Parisius/Brinkmann 1897, 116f.

53 Zitiert nach der Transkription bei Stubblebine 1979, 32.

54 Rave 1986, 26f. 


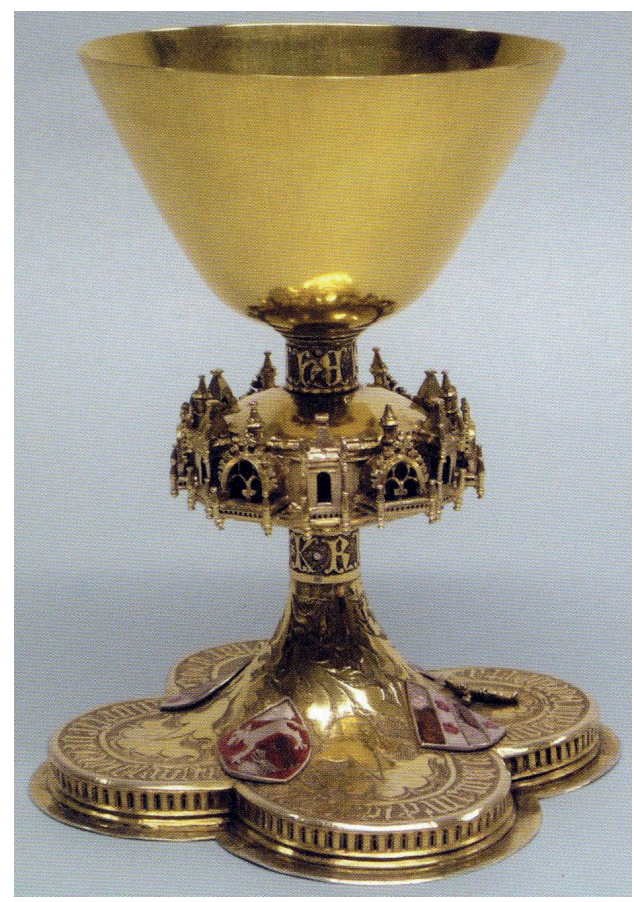

Abb. 7: Heinrich Horne, Meßkelch Anfang 15. Jahrhundert. Kloster Neuendorf

Aufgrund der Formen wie des Dekores gehört noch ein dritter Kelch in die von Heinrich Horne geschaffene Gruppe, obgleich nicht signiert. Der Kelch (Anfang 15. Jahrhundert) ist heute in Kloster Diesdorf, stammt aber gleichfalls aus Kloster Althaldensleben, gestiftet von Godeleve von Bartensleben: „hv(n)c calice(m) de(di)t go // de(lev)e vxor bvrch(a)rdi // de b(er)te(n)sle(ve) ad clav // str(vm) halde(n)sle(ve) p(ro) memo(ria)“; der Fuß trägt die Wappen der Familien Bartensleben und Alvensleben. ${ }^{55}$

Vor dem Hintergrund der vorgestellten Tafelbilder der Gregorsmesse und in Zusammenschau mit den zitierten Kelchinschriften sei die oben geäußerte These erneut aufgegriffen: Liegt die mögliche Erklärung dafür, dass der Großteil der Kelche mit Erlösungsbitten an die Zelebranten in der Zeit nach ca. 1350 entstand, vielleicht darin, dass erst im Verlauf der zweiten Hälfte des 14. Jahrhunderts die eucharistischen Gaben von Brot und Wein im Denken der Zeit um die Erlösung der armen Seelen gleichziehen?

Allerdings führt die Spur zeitlich weiter zurück, denn Jakobus de Voragine überliefert in seiner Legenda Aurea eine Kelchdonation Kaiser Heinrichs II., welche des Kaisers Seele um Haaresbreite vor der Hölle rettete. Heinrich hatte seine Gemahlin Kunigunde des Ehebruchs bezichtigt; die Kaiserin indes war unschuldig, denn sie

55 Seyderhelm 2001a, 260-261; Seyfried 1993, 19. 
bestand die geforderte Probe, über glühende Pflugscharen zu gehen. Damit hatte Heinrich Schuld auf sich geladen, und als er starb, kamen die Teufel, um seine Seele $\mathrm{zu}$ holen. Doch rettete ihn die einst gemachte Kelchdonation; so berichtet einer der Teufel einem Einsiedler vom misslungenen Plan: „Denn“, schreibt Jakobus de Voragine,

da der falsche Verdacht um sein Gemahl mit anderen Sünden auf die Schale der Waage ward gelegt und die guten Werke auf die andere, da trat dieser gebrannte Laurentius hinzu und tat einen schweren goldenen Krug auf die Waagschale, dass sie sank; da wir schon gemeint hatten, wir würden den Kaiser haben. Da brach ich in großem Zorn von dem Krug ein Ohr ab. Was aber der Teufel ein Krug nannte, das war der Kelch, den der Kaiser der Kirche von Eichstädt Sanct Laurenzio zu Ehren hatte lassen machen, da er den Heiligen in sonderlicher Andacht hielt, und hatte der Kelch von großer Schwere wegen zwei Ohren. ${ }^{56}$

Folglich handelte es sich um einen Doppelhenkelkelch von Typus des calix maior bzw. calix ministerialis für die Kelchkommunion; verwiesen sei hier auf die beeindruckende Gruppe an Beispielen, die Victor H. Elbern um den Kelch aus Ardagh (Dublin, National Museum of Ireland), jenen des Gauzelinus (Nancy, Domschatz) sowie die beiden aus Stift Wilten und aus dem Kloster Sankt Peter zu Salzburg scharte (beide Wien, Kunsthistorisches Museum).$^{57}$

Was die Heinrichsvita bei Jakobus de Voragine betrifft, so verdanke ich Tino Licht (Heidelberg) folgenden Hinweis: Um 1146 entsteht zu Bamberg im Zuge der Heiligsprechung Heinrichs II. eine Vita desselben; deren 18. Kapitel schildert die bereits zitierte Seelenwaage, bei welcher der hl. Laurentius jenen Kelch - hier aber von Kaiser Heinrich in den Dom von Merseburg gestiftet - rettend in die Waagschale legt. 1004 hatte Heinrich II. das Bistum Merseburg restituiert, das unter Otto II. kassiert worden war. Heinrich beschenkte die Bischofskirche reich; unter den kaiserlichen Gaben listet Thietmar von Merseburg (Chronik, VI, 102) auch jenen Kelch auf. ${ }^{58}$

Es ist jedoch ganz unnötig - das sei an dieser Stelle unterstrichen - eine Kelchdonation auf den Gebrauch während individuell gestifteter Seelenmessen einzuengen, denn jede Messe beinhaltet ein sich an die Wandlung anschließendes Memento für die Verstorbenen - meist allerdings ein kollektives. ${ }^{59}$ Vor dem geschilderten Hintergrund betrachtet, entfalten somit Kelchdonationen zusammen mit den angebrachten Bittinschriften eine gleichsam doppelte Wirkung bei der Errettung der Seele des Stifters.

Doch zurück $\mathrm{zu}$ den fürbittenden Inschriften und $\mathrm{zu}$ deren Position samt der damit verbundenen Sichtbarkeit.

56 Jacobus de Voragine 2014, 440-441. Jezler/Sibillano 1994, 221.

57 Elbern 1963, 21, 23-25, 28f., 34-41, 168-171.

58 Stumpf 1999, 303-305. Mein herzlicher Dank geht an Tino Licht, Universität Heidelberg, für sämtliche inhaltlichen wie bibliographischen Hinweise.

59 Zum sich an die Wandlung anschließenden Memento für die Verstorbenen siehe Jungmann, II, 1962, 295-308. 


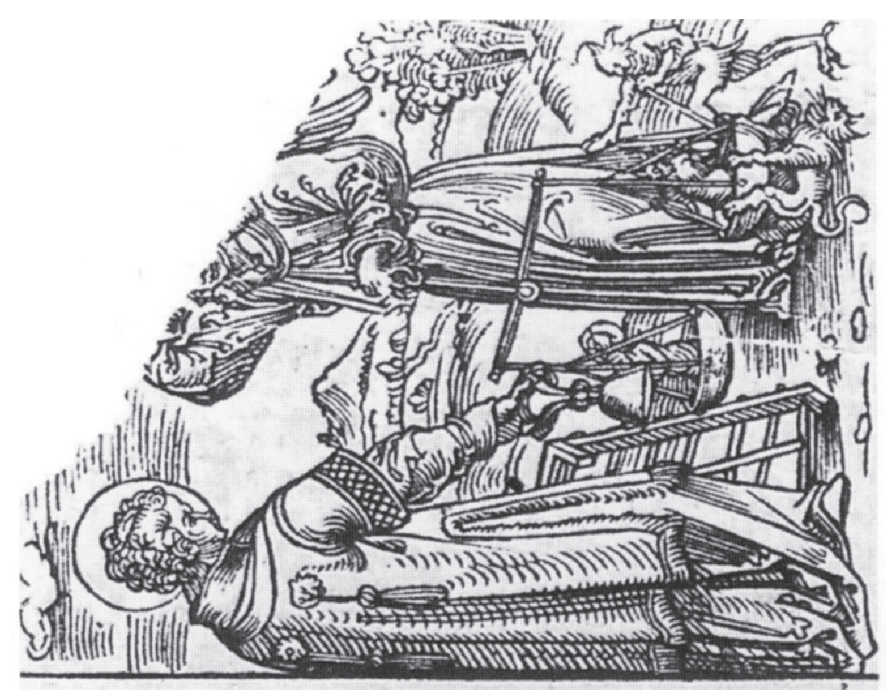

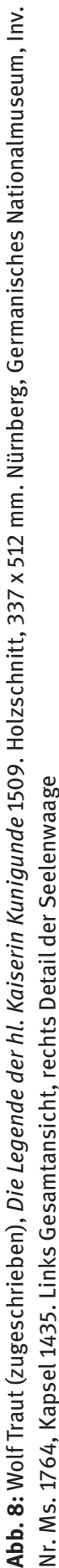

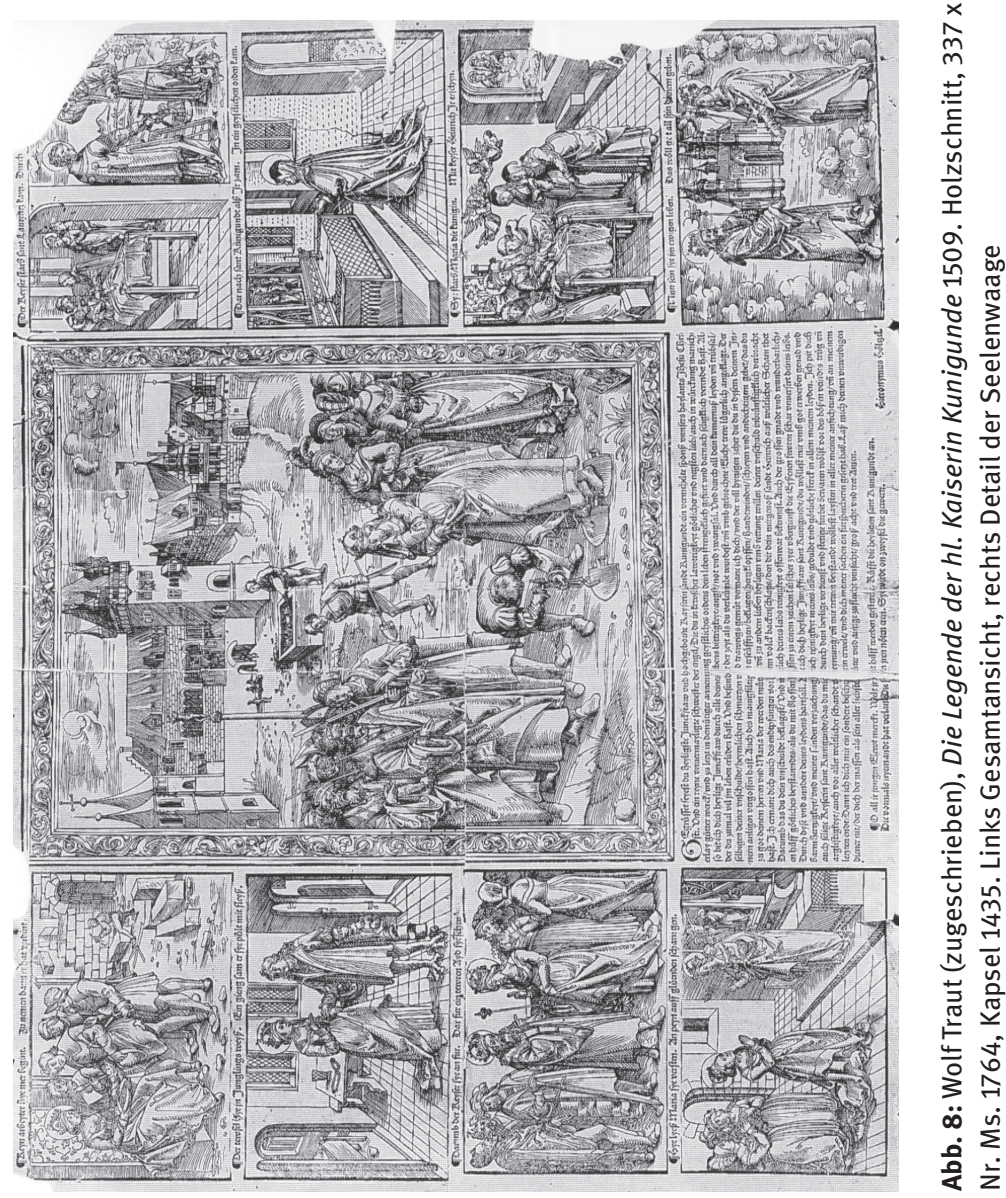




\section{Die Sichtbarkeit der Inschrift und ihr Verhältnis zu Adressat und Wirkung}

Bei den bereits vorgestellten Patenen aus Stûma und Resafa (Abb. 1) sowie bei den beiden Pyxiden zu Salzwedel (Abb. 2) und Osnabrück wollten die ,Worte' buchstäblich ,begriffen' werden und brachten so ihre Botschaften den Zelebranten nahe. Bei den Kelchen dagegen ist die Sache noch raffinierter, denn die mahnenden Worte stehen entweder auf der Zarge des Fußes oder auf dem Rand über derselben; somit passieren die eingravierten Worte bei der Elevation des Kelches unweigerlich den Blick des Priesters und appellieren unübersehbar an denselben zur individuellen Fürbitte für das Seelenheil des Wohltäters. Ganz in diesem Sinne wendet sich die Inschrift des Kelches zu St. Marien in Weißenfels (um 1400) direkt an den zelebrierenden Priester und mahnt ihn: „hoc tv cvm calice qvi missam vis celebrare sis memor hinrici fvndator(is)". ${ }^{60}$ Ähnlich appellieren die merkwürdigerweise identischen Inschriften der Kelche (um 1400) in der Marienkirche zu Uelzen und aus der 1860/61 niedergelegten Lambertikirche zu Lüneburg: „Missam qui dicis in honore Dei Genetricis - Hoc vas pro dante tu post orabis et ante. Amen“. ${ }^{61}$

Daran anknüpfend läuft eine verhältnismäßig dichte Abfolge an Beispielen bis in die Zeit der Reformation: Den Anfang macht die Inschrift des Kelches (Anfang 15. Jahrhundert) der Petrikirche zu Rostock: „Kord proyte vnde alheit zyne husvrowe gheven dessen kelk dorch got, o(ra) p(ro) eis. “62 Ihr folgt die des Kelches (dat. 1468) der Peterskirche in Buxtehude: „Iste calix spectat ad commendas a magistro gherardo halepage anno domini MCCCCLXVIII fundatas • orate pro donatore“ ${ }^{63}$ zusammen mit der des 1478 in die Klosterkirche zu Amelungsborn gestifteten Kelches: „orate p(ro) iohane abb(at)e an(n)o m lxxciii“; es handelt sich um den 27. Abt von Amelungsborn, Johannes VI. von Dassel, der von 1477 bis zu seinem Tode am 5. Juli 1483 amtierte. ${ }^{64}$ Ähnlich lautet der Wunsch auf dem Kelch aus der zweiten Hälfte des 15. Jahrhunderts aus Rø auf der Insel Bornholm „biddet got vor bernt zegevrit, vor sine vrowe, vor er beyder geslechte“. ${ }^{65}$

Daran schließt sich die Inschrift des im Jahre 1500 gestifteten Exemplars der Salzwedeler Marienkirche an: „p(ro) me(m)oria d(omi)ni mathie et iochim schulteti

60 „Willst Du mit diesem Kelch die Messe zelebrieren, gedenke Heinrich dem Stifter“; Jäger 2005, 26f., Nr. 28; Ellwardt 2001, 238f., hier in die Mitte bis 2. Hälfte 14. Jahrhundert datiert; Ellwardt 2004, 231-233.

61 „Liest Du die Messe zu Ehren der Gottesgebärerin, dann bete zuvor und danach für den, der dies Gefäß stiftete“; Braun 1932, 172; Mithoff 1877, 150f. (Kelch aus der ehemaligen Lamberti-Kirche Lüneburg), 257 (Kelch der Marienkirche Uelzen).

62 Schlie, I, 1896, 120 mit Abb.; Braun 1932, 171f.; Fritz 1966, 176, 533 mit Katalognummer 635.

63 Mithoff 1878, 32; „dieser Kelch wird den Vermächtnissen von Meister Gerhard Halepage verdankt, im Jahre des Herrn 1468 getätigt, betet für den Wohltäter“.

64 Lampe/Willing 2012, 78-81, Nr. 29.

65 Fritz 1982, 92. 
$\mathrm{f}\left(\right.$ rat)res et eorv(m) pare(n)tes o(r)ate deu(m) p(ro) en(sie) (= eis) datu(m) $\mathrm{m}^{\circ}$ ccccc $^{\circ \text { “ } ; 66}$ die Reihe beschließt die Bitte des 1507 datierten Kelches in der Kirche zu West Drayton in England: „Orate pro animabus Johis Porpyll et Johannae uxoris“. ${ }^{67}$

\section{Präsenz ohne Sichtbarkeit}

Alle bisherigen Beispiele eint, dass zwar die Sichtbarkeit jedoch nicht unbedingt die spontane Lesbarkeit der Schrift garantiert ist, damit ihre Wirkung sich entfalte und die Adressaten während der Messe und der Wandlung der eucharistischen Gaben erreicht werden.

Doch sind es die Kelche selbst, die Hinweise auf eine weitere Sinnschicht der Präsenz des Stifters in seiner Inschrift geben, und zwar einer, welche um das Wissen um die Präsenz kreist, ja sogar jenseits der visuellen Präsenz liegt und somit wichtiger ist als die visuelle Präsenz selbst: Das belegt eine Gruppe von Kelchen, die alle Bittinschriften unten auf ihren Standflächen tragen, infolgedessen auch bei der Elevation schwerlich sichtbar und schon gar nicht zu entziffern sind. Das bedeutet, Präsenz entsteht keinesfalls ausschließlich durch Sichtbarkeit. Dass hier der Gedanke der Humilitas von Seiten des Stifters dahinterstehen könnte, scheint naheliegend, wäre aber noch zu klären. Zeitlich bildet der bereits vorgestellte Kelch (Anfang 15. Jahrhundert, Medaillons 2. Viertel 14. Jahrhundert) der Marienkirche zu Gardelegen den Anfang; die unten in die Standfläche des Fußes eingravierten Worte mahnen: „orate pro hinriko horne aurifabro“. ${ }^{6}$

Fast flehentlich lautet die Bitte eines 1443 datierten Kelches aus Sankt Nikolai zu Nordhausen, ebenfalls quasi unsichtbar unten in die Standfläche eingraviert: „anno $\mathrm{d}\left(\right.$ omini) mcccc $^{\circ}$ xliii istum calicem dedit joh(ann)es schutcze et $†$ katerina ejus vxor in remedium a(n)imarum eorv(n)dem“. ${ }^{69}$ Zum Schluss das dritte und prächtigste Stück der kleinen Gruppe: So heißt es unten auf dem rein goldenen Kelch, 1504 vom Hamburger Goldschmied Hinrick Rentzel getrieben und von den Brüdern Johannes und Heinrick von Schauenburg ins Zisterzienserinnenkloster Uetersen (Kreis Pinneberg) gestiftet (Schleswig, Landesmuseum, Schloss Gottorf): ,anno dni cxv iii iohannes schouwenborch et frater hinrick eivs dederunt hu(n)c calicem aureum orate pro eis". 70

66 „zum Gedächtnis des Herrn Matthias und Joachim Schultheis, Brüder, und deren Eltern, betet zu Gott für sie; gestiftet im Jahre 1500“; Bergemann 2001, 302; Fischer 1990, 80 mit Abbildung; Dehio 1974, 350.

67 Braun 1932, 171.

68 Heidemann 2001b, 256-258; Dehio 1974, 10; Parisius/Brinkmann 1897, 75f.

69 „Im Jahre des Herrn 1443 schenkte diesen Kelch Johannes Schütze gemeinsam mit seiner Ehefrau Katharina zu ihrer beiden Seelenheil“; Seyderhelm 2001b, 279-280; Schmidt 1887, 134.

70 Fritz 1982, 304, 340-341, Abb. 852. 


\section{Inschriften und deren Metamorphose: Weihrauchfässer}

Bei keinen liturgischen Geräten erfahren Worte aber eine vergleichbare Verwandlung wie die Worte der Bittinschriften auf Weihrauchfässern, denn es sind die Weihrauchschwaden jener während den Messen vollzogenen Inzensierungen, die Fürbitten und Gebete zu Gott tragen, steht doch der Weihrauch für das zu Gott aufsteigende Gebet gemäß Psalm 141 und Offb 8,3f. ${ }^{71}$ Auf dem bronzenen Weihrauchfass des Reiner (2. Viertel 12. Jahrhundert. Lille, Musée des Beaux-Arts) lautet darum die Inschrift: „HOC EGO REINER(US) DO SIGNU(M) QUID MICHI VESTRIS / EXEQUIAS SIMILES DEBETIS MORTE POTITO / ET REOR ESSE PRESES V(E)RAS TIMIAMATA XP(IST)O“; ;2 und auf dem Weihrauchfass des Gozbert (12. Jahrhundert. Trier, Domschatz): „HAEC TV QVESO VIDENS GOZBERTUS SIT PETE VIVENS.“"73 Das bislang jüngste Beispiel bildet das Rauchfaß des Kopenhagener Nationalmuseums, das aus der Kirche von Ulbølle stammt. Im 13. Jahrhundert goss es Meister Jacob Røld (Magister Rufus) aus Svendborg. Seine Inschrift - teilweise Runentext in altdänischer Lautsprache, teilweise in Latein - lautet verdeutscht: „Meister Jakob Rot machte mich. Er gab mich an dem ... [für die Seele] seiner geliebten Hausfrau Meret. Gott segne den, der mich machte. Ave Maria gratia plena dominus tecum benedicta tu in mulieribus. Ebmyæflnhi" ${ }^{74}$ Damit verschwinden Fürbittinschriften auf Weihrauchfässern, denn für die hoch- und spätgotischen Stücke sind bislang keine überliefert. Dass dieses Verschwinden ins 13. Jahrhundert fällt, lässt zwar aufhorchen, doch wundert es wenig, denn das Abendland konzentriert sich seit dieser Zeit auf die eucharistische Frömmigkeit und huldigt dieser überschwänglich - wie die vergangenen Abschnitte zeigten - auf Patenen, Monstranzen, Pyxiden und Kelchen.

Zusammenfassend lässt sich sagen, dass bei Fürbittinschriften auf vasa sacra wie non sacra die Stifter ,expressis verbis‘ den Zelebranten, die mit den Gefäßen in Kontakt kommen, einen Wunsch auftragen. Er gilt der Fürbitte, dem ewigen Gedenken und

71 Pfeifer 2008, 76-79, 85f., 90-98; Westermann-Angerhausen 2014, 28f., 84-86.

72 „Ich, Reiner, mache folgendes Zeichen: Wozu schuldet Ihr mir, wenn ich den Tod erreicht habe, eine Beisetzungsfeier der euren gleich? Ich glaube zudem, dass echte Gebete Weihrauch für Christus sind“; Übersetzung und Sachverhalt nach Legner 2009, 327f. Westermann-Angerhausen 2014, 28f., 205-207, Nr. II p 2.

73 „Wenn Du dieses siehst, bete, so bitte ich Dich, dass Gozbertus das Leben beschieden sei“; Übersetzung und Sachverhalt nach Legner 2009, 327f.; Westermann-Angerhausen 2014, 28, 214-223, Nr. II q; Übersetzung nach Westermann-Angerhausen: „Du, der Du dieses siehst, wer Du auch seiest, bete, daß Gozbertus lebe.“.

74 „, mæ.......p : gørpæ : mik : han : gifær : mik : pæn $1+$ hæl : rik • k xxxxxx tkr • kær : hus : rkø : mærutæ : siul : gup : sihnn : 1 paen : mik : kørpæ : auemari agrsia : plena : somin : tikum $\bullet 1$ bentik : tatu : in : mleri : ibus : ebmyæflnhi“; sämtliche Informationen nach Westermann-Angerhausen 2014, 316f., Nr. IV c 1. 
der Erlösung innerhalb des kontinuierlich wiederkehrenden Wunders der Wandlung der eucharistischen Gaben; die in den vergangenen Abschnitten präsentierten Beispiele sind überwiegend auf Latein und meist im Auftrag von Laien eingraviert. Neben der Bitte um Vergebung und Erlösung spielt der Stellvertretercharakter der Worte auf Patenen, Pyxiden oder Kelchen eine zentrale Rolle, denn die Inschriften ermöglichen den Laienstiftern eine unmittelbare Teilnahme am eucharistischen Opfer, von dem sie leiblich auf Abstand gehalten werden. In diesem Falle liegt der Schwerpunkt zunächst auf der Sichtbarkeit der Schrift und erst in zweiter Linie auf deren leichter Lesbarkeit.

Ferner entfalten die Fürbittinschriften bei eucharistischen Monstranzen eine Transzendenz und bei Weihrauchfässern erfahren sie eine Metamorphose. Bei den eucharistischen Monstranzen profitiert die Bittinschrift von der damals unumstößlichen Ansicht, dass sie gekoppelt an das göttliche, von der gewandelten Hostie ausgehenden lumen unmerklich in die Seele der Andächtigen fällt und mit deren Gebeten Erhörung bei Gott findet.

Bei der Metamorphose der Inschriften auf den Weihrauchfässern steht der feste Glaube dahinter, dass die Weihrauchschwaden jener während den Messen vollzogenen Inzensierungen die Fürbitten und Gebete zu Gott tragen, denn der Weihrauch steht für das zu Gott aufsteigende Gebet. Sämtliche dargelegten Sinnschichten einigt dann aber wieder die Tatsache, dass nämlich das inschriftlich perpetuierte Anliegen der Stifter seine Wirkung keineswegs aus einer leichten Lesbarkeit generiert, sondern aus seiner Präsenz innerhalb eines rituell korrekten Kontextes.

\section{Literaturverzeichnis}

Andersson, Aron (1956), Silberne Abendmahlsgeräte in Schweden aus dem XIV. Jahrhundert, 2 Bände, Uppsala.

Andrae, Katharina (2001), Katalogbeitrag „Nr. 66 Kelch“, in: Goldschmiedekunst des Mittelalters im Gebrauch der Gemeinden über Jahrhunderte bewahrt (Katalog zur Ausstellung im Dom zu Magdeburg, in der Stiftskirche Quedlinburg und in der Stadtkirche Wittenberg 2001-2002), hg. v. Bettina Seyderhelm, Dresden, $258 f$.

Andrae, Katharina (2004), „Nr. 18 Kelch“, in: Goldschmiedekunst des Mittelalters aus evangelischen Kirchen Ostdeutschlands (Katalog zur Ausstellung The National Museum of Western Art in Tokyo, 29. Juni-15. August 2004), hg. von Bettina Seyderhelm u. Mikinosuke Tanabe, Tokyo, 146-148.

Bergdolt, Klaus (1988), Der dritte Kommentar Lorenzo Ghibertis. Naturwissenschaften und Medizin in der Kunsttheorie der Frührenaissance (VCH, Acta Humaniora), Weinheim.

Bergemann, Uta-Christiane (2001), „Nr. 96 Kelch“, in: Goldschmiedekunst des Mittelalters im Gebrauch der Gemeinden über Jahrhunderte bewahrt (Katalog zur Ausstellung im Dom zu Magdeburg, in der Stiftskirche Quedlinburg und in der Stadtkirche Wittenberg 2001-2002), hg. von Bettina Seyderhelm, Dresden, 302.

Birgitta (2008), The Revelations of St. Birgitta of Sweden, volume 2. Liber Caelestis, Books IV-V,trans. by Denis Searby with Introductions and Notes by Bridget Morris, Oxford. 
Birgitta (2015), The Revelations of St. Birgitta of Sweden, volume 4. The Heavenly Emperor's Book to Kings, The Rule, and Minor Works trans. by Denis Searby with Introductions and Notes by Bridget Morris, Oxford.

Braun, Joseph (1932), Das christliche Altargerät in seinem Sein und in seiner Entwicklung, München. Browe, Peter (1933), Die Verehrung der Eucharistie im Mittelalter, München.

Degen, Rainer (1990), „III. Inschriften, 3. Die Patene“, in: Thilo Ulbert (Hg.), Der kreuzfahrerzeitliche Silberschatz aus Resafa-Sergiupolis (Deutsches Archäologisches Institut, Resafa III), Mainz, 68-74.

Dehio, Georg (1974), Handbuch der deutschen Kunstdenkmäler. Der Bezirk Magdeburg (Neubearbeitung Beate Becker, Gerda Herrmann, Christa Stepansky, Heinrich Trost), hg. von Edgar Lehmann, Berlin.

Elbern, Victor H. (1963), „Der eucharistische Kelch im frühen Mittelalter“, Zeitschrift des Deutschen Vereins für Kunstwissenschaft XVII, 1963, 1-76, 117-188.

Ellwardt, Kathrin (2001), „Nr. 51 Kelch“, in: Goldschmiedekunst des Mittelalters im Gebrauch der Gemeinden über Jahrhunderte bewahrt (Katalog zur Ausstellung im Dom zu Magdeburg, in der Stiftskirche Quedlinburg und in der Stadtkirche Wittenberg 2001-2002), hg. von Bettina Seyderhelm, Dresden, $238 \mathrm{f}$.

Ellwardt, Kathrin (2004), „Nr. 61 Kelch“, in: Goldschmiedekunst des Mittelalters aus evangelischen Kirchen Ostdeutschlands (Katalog zur Ausstellung The National Museum of Western Art in Tokyo, 29. Juni-15. August 2004), hg. von Bettina Seyderhelm u. Mikinosuke Tanabe, Tokyo, 231-233.

Fischer, Peter (1990), Denkmale des Kreises Salzwedel, hg. von Freilichtmuseum Diesdorf, Diesdorf. Franz, Adolph (1902), Die Messe im deutschen Mittelalter, Freiburg im Breisgau.

Frese, Tobias (2013), Aktual- und Realpräsenz. Das eucharistische Christusbild von der Spätantike bis ins Mittelalter, Berlin.

Fritz, Johann Michael (1966), Gestochene Bilder. Gravierungen auf deutschen Goldschmiedearbeiten der Spätgotik (Beihefte der Bonner Jahrbücher 20), Köln, Graz.

Fritz, Johann Michael (1982), Goldschmiedekunst der Gotik in Mitteleuropa, München.

Gärtner, Claudia (2007), „Die ,Gregorsmesse’ als Bestätigung der Transsubstantiationslehre? Zur Theologie des Bildsujets“, in: Andreas Gormans u. Thomas Lentes (Hgg.), Das Bild der Erscheinung. Die Gregorsmesse im Mittelalter (Kultbild. Visualität und Religion in der Vormoderne, Bd. III, hg. v. Thomas Lentes), Berlin, 125-153.

Grieshaber, Franz Karl (1844), Deutsche Predigten des XIII. Jahrhunderts, 2. Abteilung, Hom. 613 m-I, Stuttgart.

Habenicht, Georg (2016), Das ungefasste Altarretabel. Programm oder Provisorium (Studien zur internationalen Architektur- und Kunstgeschichte 144), Petersberg.

Heidemann, Susanne (2001a), „Nr. 27 Pyxis“, in: Goldschmiedekunst des Mittelalters im Gebrauch der Gemeinden über Jahrhunderte bewahrt (Katalog zur Ausstellung im Dom zu Magdeburg, in der Stiftskirche Quedlinburg und in der Stadtkirche Wittenberg 2001-2002), hg. von Bettina Seyderhelm, Dresden, 204.

Heidemann, Susanne (2001b), „Nr. 65 Kelch“, in: Goldschmiedekunst des Mittelalters im Gebrauch der Gemeinden über Jahrhunderte bewahrt (Katalog zur Ausstellung im Dom zu Magdeburg, in der Stiftskirche Quedlinburg und in der Stadtkirche Wittenberg 2001-2002), hg. von Bettina Seyderhelm, Dresden, 256-258.

Heidemann, Susanne (2004), „Nr. 7 Pyxis“, in: Goldschmiedekunst des Mittelalters aus evangelischen Kirchen Ostdeutschlands (Katalog zur Ausstellung The National Museum of Western Art in Tokyo, 29. Juni-15. August 2004), hg. von Bettina Seyderhelm u. Mikinosuke Tanabe, Tokyo, $120 f$. 
Jackson, Charles James (1967), An Illustrated History of English Plate, Bd. I (London 1911), Nachdruck, London.

Jäger, Franz (2005), Die Inschriften des Landkreises Weißenfels (Die deutschen Inschriften 62, Leipziger Reihe 1), Wiesbaden.

Jakobus de Voragine $\left(2014^{16}\right)$, Legenda Aurea, übers. von Carl Benz, mit einem Nachwort v. Walter Berschin, Gütersloh.

Jezler, Jezler $\left(1994^{2}\right)$, „Jenseitsmodelle und Jenseitsvorsorge - eine Einführung“, in: Himmel Hölle - Fegefeuer. Das Jenseits im Mittelalter (Katalog zur Ausstellung des Schweizerischen Landesmuseums in Zürich, 4. März-29. Mai 1994 und des Schnütgen-Museums in Köln, 21. Juni-28. August 1994), hg. von der Gesellschaft für das Schweizerische Landesmuseum, München, 13-26.

Jezler, Peter (Ikonographie)/Konrad, Konrad (stilistische Einordnung) (1994²), „97. Kopfüber schweben Engel ins Fegefeuer hinab, um mit Almosen und Hostien Arme Seelen zu retten“, in: Himmel - Hölle - Fegefeuer. Das Jenseits im Mittelalter (Katalog zur Ausstellung des Schweizerischen Landesmuseums in Zürich, 4. März-29. Mai 1994 und des Schnütgen-Museums in Köln, 21. Juni-28. August 1994), hg. von der Gesellschaft für das Schweizerische Landesmuseum, München, $290 f$.

Jezler, Peter/Sibillano, Pasquale $\left(1994^{2}\right)$, „42. Eine Kelchdonation kann die Seele vor der Hölle retten“, in: Himmel - Hölle - Fegefeuer. Das Jenseits im Mittelalter (Katalog zur Ausstellung des Schweizerischen Landesmuseums in Zürich, 4. März-29. Mai 1994 und des SchnütgenMuseums in Köln, 21. Juni-28. August 1994), hg. von der Gesellschaft für das Schweizerische Landesmuseum, München, 221.

Jungmann, Josef Andreas (19625), Missarum Sollemnia. Eine genetische Erklärung der Römischen Messe. Bd. 2, Opfermesse, Wien/Freiburg/Basel.

Kisters, Fritz (1994²), „98. In der Gregorsmesse fließt das Blut Christi über den Altar und öffnet den Armen Seelen den Weg in den Himmel“, in: Himmel - Hölle - Fegefeuer. Das Jenseits im Mittelalter (Katalog zur Ausstellung des Schweizerischen Landesmuseums in Zürich, 4. März-29. Mai 1994 und des Schnütgen-Museums in Köln, 21. Juni-28. August 1994), hg. von der Gesellschaft für das Schweizerische Landesmuseum, München, 294.

Kratzke, Christine (2004), Das Zisterzienserkloster Dargun in Mecklenburg-Vorpommern (Studien zur internationalen Architektur- und Kunstgeschichte 25), Petersberg.

Laabs, Annegret (2000), Malerei und Plastik im Zisterzienserorden. Zum Bildgebrauch zwischen sakralem Zeremoniell und Stiftermemoria 1250-1430 (Studien zur internationalen Architekturund Kunstgeschichte 8), Petersberg.

Lampe, Jörg H./Willing, Meike (2012), Die Inschriften des Landkreises Holzminden (Die deutschen Inschriften 83, Göttinger Reihe 15), Wiesbaden.

Lanz, Hanspeter (1994²), „43/44. Ritter Konrad von Wolfurt stellt für sich, seine Vorfahren und all jene, die er verletzt oder getötet hat, ein Seelgerät bereit. Dazu gehört auch ein Kelch. Alles wird in einer Urkunde verzeichnet“, in: Himmel - Hölle - Fegefeuer. Das Jenseits im Mittelalter (Katalog zur Ausstellung des Schweizerischen Landesmuseums in Zürich, 4. März-29. Mai 1994 und des Schnütgen-Museums in Köln, 21. Juni-28. August 1994), hg. von der Gesellschaft für das Schweizerische Landesmuseum, München, $222 \mathrm{f}$.

Legner, Anton (2009), Der artifex. Künstler im Mittelalter und ihre Selbstdarstellung. Eine illustrierte Anthologie, Köln.

Mithoff, H. Wilhelm H. (1877), Kunstdenkmäler im Hannoverschen. Bd. IV: Fürstenthum Lüneburg, Hannover.

Mithoff, H. Wilhelm H. (1878), Kunstdenkmäler im Hannoverschen. Bd. V: Herzogtümer Bremen und Verden mit dem Lande Hadeln, Grafschaften Hoya und Diepholz, Hannover. 
Omont, Henri (1901), „Inventaires du Trésor et des Objets précieux conservés dans l’Église de l’Abbaye de Saint-Denis en 1505 et 1739“, Mémoires de la Société de l'Histoire de Paris et de I'lle-de-France XXVIII, 163-212.

Parisius, Adolf/Brinkmann, Adolf (1897), Beschreibende Darstellung der älteren Bau- und Kunstdenkmäler der Provinz Sachsen und angrenzender Gebiete. Bd. 20: Beschreibende Darstellung der älteren Bau- und Kunstdenkmäler des Kreises Gardelegen, Halle a. d. Saale.

Pfeifer, Michael (2008²), Der Weihrauch. Geschichte-Bedeutung-Verwendung, Regensburg.

Rave, August B. (1986), Fronleichnam in Siena. Die Maestà von Simone Martini in der Sala del Mappamondo, Worms.

Schlie, Friedrich 1896, Die Kunst- und Geschichtsdenkmäler des Großherzogtums MecklenburgSchwerin. Bd. I: Die Amtsbezirke Rostock, Ribnitz, Sülze-Marlow, Tessin, Laage, Gnoien, Dargun, Neukalen, Schwerin.

Schmidt, Julius (1887), Beschreibende Darstellung der älteren Bau- und Kunstdenkmäler der Stadt Nordhausen (Beschreibende Darstellung der älteren Bau- und Kunstdenkmäler der Provinz Sachsen und angrenzender Gebiete XI: Die Stadt Nordhausen), Halle a. d. Saale.

Seyderhelm, Bettina (2001a), „Nr. 67 Kelch“, in: Goldschmiedekunst des Mittelalters im Gebrauch der Gemeinden über Jahrhunderte bewahrt (Katalog zur Ausstellung im Dom zu Magdeburg, in der Stiftskirche Quedlinburg und in der Stadtkirche Wittenberg 2001-2002), hg. von Bettina Seyderhelm, Dresden, 260f.

Seyderhelm, Bettina (2001b), „Nr. 76 Kelch“, in: Goldschmiedekunst des Mittelalters im Gebrauch der Gemeinden über Jahrhunderte bewahrt (Katalog zur Ausstellung im Dom zu Magdeburg, in der Stiftskirche Quedlinburg und in der Stadtkirche Wittenberg 2001-2002), hg. von Bettina Seyderhelm, Dresden, $279 f$.

Seyfried, Peter (1993), Kloster Diesdorf in der Altmark (Große Baudenkmäler 463), München/Berlin.

Skubiszewski, Piotr (2003), „L“iconographie de la patène et du calice du frère Hugo“, in: Autour de Hugo d'Oignies (Katalog der Ausstellung Musée des Arts Anciens du Namurois in Namur, 29. Mai-30. November 2003), hg. von Robert Didier u. Jacques Toussaint, Namur, 99-123.

Stubblebine, James H. (1979), Duccio di Buoninsegna and His School, Bd. I, Princeton (NJ).

Stumpf, Marcus (1999), Die Vita sancti Heinrici regis et confessoris und ihre Bearbeitung durch den Bamberger Diakon Adelbert, Hannover.

Tripps, Johannes $\left(2000^{2}\right)$, Das handelnde Bildwerk in der Gotik. Forschungen zu den Bedeutungsschichten und der Funktion des Kirchengebäudes und seiner Ausstattung in der Hoch- und Spätgotik, Berlin.

Westermann-Angerhausen, Hiltrud (2014), Mittelalterliche Weihrauchfässer von 800 bis 1500 (Denkmäler deutscher Kunst, hg. v. Deutschen Verein für Kunstwissenschaft. Bronzegeräte des Mittelalters, begr. v. Otto von Falke und Erich Meyer, fortgeführt v. Peter Bloch, hg. v. Rainer Kahsnitz 7), Petersberg. 


\section{Abbildungsnachweise}

Abb. 1: Reproduktion nach Degen 1990, Foto Helmuth Nils Loose (Resafa-Archiv)

Abb. 2: Reproduktion nach Heidemann 2004, Foto Ulrich Sieblist

Abb. 3: Reproduktion nach Jezler/Konrad 1994, Foto Zürich, Schweizerisches Landesmuseum

Abb. 4: Reproduktion nach Skubiszewski 2003, Foto IRPA/KIK, Bruxelles

Abb. 5: Reproduktion nach Gärtner 2007

Abb. 6: Reproduktion nach Kister 1994, Foto Sammlung Heinz Kisters

Abb. 7: Reproduktion nach Andrae 2004, Foto Ulrich Ahrenmeier, Hannover

Abb. 8: Reproduktion nach Jezler/Sibillano 1994, Foto Nürnberg, Germanisches Nationalmuseum 
